\title{
Altered elF6 and Dicer expression is associated with clinicopathological features in ovarian serous carcinoma patients
}

Richard J Flavin ${ }^{1}$, Paul C Smyth ${ }^{1}$, Stephen P Finn ${ }^{2}$, Alexandros Laios ${ }^{3}$, Sharon A O’Toole ${ }^{3}$, Ciara Barrett ${ }^{1}$, Martina Ring ${ }^{1}$, Karen M Denning ${ }^{1}$, Jinghuan Li $^{1}$, Sinead T Aherne ${ }^{1}$, Natasha A Aziz ${ }^{3}$, Araibi Alhadi ${ }^{3}$, Brian L Sheppard ${ }^{3}$, Massimo Loda ${ }^{2}$, Cara Martin ${ }^{1}$, Orla M Sheils ${ }^{1}$ and John J O’Leary ${ }^{1}$

${ }^{1}$ Department of Histopathology, Trinity College Dublin, Dublin, Ireland; ${ }^{2}$ The Dana Farber Cancer Institute, Harvard Medical School, Boston, MA, USA and ${ }^{3}$ Department of Obstetrics and Gynaecology, Trinity College Dublin, Dublin, Ireland

\begin{abstract}
MicroRNAs are a group of small non-coding RNAs approximately 22 nucleotides in length. Recent work has shown differential expression of mature microRNAs in human cancers. Production and function of microRNAs require coordinated processing by proteins of the microRNA machinery. Dicer and Drosha (RNase III endonucleases) are essential components of the microRNA machinery. Recently, the ribosome anti-association factor elF6 has also been found to have a role in microRNA-mediated post-transcriptional silencing. We characterized the alterations in the expression of genes encoding proteins of microRNA machinery in ovarian serous carcinoma. Protein expression of elF6 and Dicer was quantified in a tissue microarray of 66 ovarian serous carcinomas. Dicer, Drosha and elF6 mRNA expression was analysed using quantitative reverse transcription-PCR on an independent set of $\mathbf{5 0}$ formalin-fixed, paraffin-embedded ovarian serous carcinoma samples. Expression profiles of elF6 and Dicer were correlated with clinicopathological and patient survival data. We provide definitive evidence that elF6 and Dicer are both upregulated in a significant proportion of ovarian serous carcinomas and are associated with specific clinicopathological features, most notably low elF6 expression being associated with reduced disease-free survival. The status of elF6 and proteins of the microRNA machinery may help predict toxicity and susceptibility to future interfering RNA-based therapy.

Modern Pathology (2008) 21, 676-684; doi:10.1038/modpathol.2008.33; published online 7 March 2008
\end{abstract}

Keywords: elF6; Dicer; Drosha; microRNA machinery; microRNAs; ovarian serous carcinoma

Epithelial ovarian cancer is one of the most common cancers in women and the leading cause of death from gynaecological malignancy in the western world. ${ }^{1}$ Approximately 205000 cases of ovarian cancer are diagnosed worldwide each year. ${ }^{2}$ Serous adenocarcinomas are the commonest histotype and account for $40-50 \%$ of malignant neoplasms. ${ }^{3}$ The majority of ovarian cancers present in advanced stages (III or IV) and are treated by surgery and systemic chemotherapy. Despite an initial $70-80 \%$ response rate, current therapy is frequently followed by recurrence, which is often resistant to chemotherapy, as demonstrated by $5-20 \%$ long-term survivors. ${ }^{4}$ There is an urgent need to identify

Correspondence: Dr RJ Flavin, Department of Histopathology, Phase 3 Trinity Centre for Health Sciences, St James's Hospital, James's Street, Dublin 8, Ireland.

E-mail: flavinr@tcd.ie

Received 19 December 2007; revised 15 January 2008; accepted 16 January 2008; published online 7 March 2008 novel or poorly characterized pathways to shed more insight into the tumorigenesis of this fatal neoplasm.

MicroRNAs are a class of small noncoding RNAs, approximately 22 nucleotides long, that have been found to negatively regulate gene expression. They have been found to have roles in cell growth, differentiation, apoptosis and tumorigenesis..$^{5-12}$ Recently, microRNAs were implicated in the development of ovarian cancer: 39 microRNAs are differentially regulated between tumour and normal ovarian tissue. ${ }^{13}$

MicroRNA production involves cleavage of a long nascent transcript (primary microRNA) from a 70-100 nucleotide hairpin precursor (precursor microRNA), which is in turn processed to form a microRNA duplex. One strand of this duplex is incorporated into the RISC complex where it will bind through partial sequence homology to the $3^{\prime}$ UTR of target mRNAs causing their translational repression. ${ }^{11,14,15}$ 
Collectively, the production and function of microRNA require a set of proteins known as the microRNA machinery, which includes both Dicer and Drosha. In humans, Drosha executes the initial microRNA processing step in the nucleus and the resultant precursor microRNA is exported to the cytoplasm for further processing by Dicer to generate the mature 22 nucleotide product. ${ }^{16,17}$

Recent evidence has shown that the anti-association factor eIF6 (ITGB4BP or p27BBP - a ribosome inhibitory protein known to prevent productive assembly of the $80 \mathrm{~S}$ ribosome $)^{18-21}$ is recruited by the RISC complex, enabling microRNA complexes to exert their repressive effects on protein translation. ${ }^{22}$

In this study, using real-time reverse transcription (RT)-PCR and immunohistochemistry, we examined whether the expression levels of eIF6, Dicer and Drosha, which are essential for microRNA production and function, are altered in ovarian serous adenocarcinomas. We report here for the first time an alteration in expression of eIF6 and microRNA machinery in ovarian serous adenocarcinomas and an association between low eIF6 protein expression and shortened patient disease-free survival.

\section{Materials and methods}

Ethical approval for the study was obtained from the St James's and Federation of Dublin Voluntary Hospitals Ethics Committee.

\section{Case Selection and Tumour Sample Preparation}

A total of 66 ovarian serous adenocarcinoma tumours, classified according to the FIGO system: stage (II-IV) and grade (2-3) were selected from archival formalin-fixed, paraffin-embedded tissue between the years 1991 and 2006 from St James's Hospital, Dublin. H\&E slides of all tumours were reviewed by a histopathologist (RF) and the original diagnoses were confirmed. The paraffin tissue microarrays were composed of tumours arrayed in quadruplicate and were age-matched to 40 normal ovaries containing normal ovarian surface epithelium. Table 1 lists the clinicopathological characteristics of the cases selected.

A total of 50 independent formalin-fixed, paraffinembedded tissue blocks were selected that contained over $90 \%$ tumour with contaminating stromal tissue estimated to be no more than $10 \%$, and total RNA was extracted using RecoverAll ${ }^{\mathrm{TM}}$ Total Nucleic Acid Extraction Kit (Ambion Ltd, Cambridgeshire, UK) following the manufacturer's protocol. RNA quantity was assessed using a Nanodrop ${ }^{\circledR}$ ND-1000 Spectrophotometer (Wilmington, USA). Reverse transcription was carried out at $25^{\circ} \mathrm{C}$ for $10 \mathrm{~min}$, $37^{\circ} \mathrm{C}$ for $120 \mathrm{~min}, 85^{\circ} \mathrm{C}$ for $5 \mathrm{~s}$ followed by incubation at $4^{\circ} \mathrm{C}$, using $20 \mathrm{ng}$ total RNA and the High-Capacity cDNA Reverse Transcription Kit (Applied Biosystems, Foster City, CA, USA).
Table 1 Relationship between expression levels of eIF6, Dicer and various clinicopathological characteristics

\begin{tabular}{|c|c|c|c|c|c|c|c|c|}
\hline \multirow[t]{2}{*}{ Characteristics } & \multirow[t]{2}{*}{ Cases } & \multicolumn{3}{|c|}{ eIF6 } & \multirow[t]{2}{*}{ Cases } & \multicolumn{3}{|c|}{ Dicer } \\
\hline & & High & Low & $\mathrm{P}^{*}$ & & High & Low & $\mathrm{P}^{*}$ \\
\hline \multicolumn{9}{|l|}{ Age } \\
\hline$\leq 55$ & 14 & 13 & 1 & 0.4 & 30 & 25 & 5 & 0.09 \\
\hline$>55$ & 50 & 42 & 8 & & 34 & 22 & 12 & \\
\hline \multicolumn{9}{|l|}{ Stage } \\
\hline II & 9 & 8 & 1 & 0.56 & 9 & 7 & 2 & 0.59 \\
\hline III-IV & 47 & 38 & 9 & & 48 & 33 & 15 & \\
\hline Unknown & 8 & & & & 7 & & & \\
\hline \multicolumn{9}{|l|}{ Grade } \\
\hline 2 & 28 & 23 & 5 & 0.87 & 29 & 23 & 6 & 0.23 \\
\hline 3 & 36 & 29 & 7 & & 32 & 21 & 11 & \\
\hline Unknown & & & & & 3 & & & \\
\hline \multicolumn{9}{|c|}{ Lymph node metastases } \\
\hline $\mathrm{Y}^{\mathrm{T}}$ & 14 & 9 & 5 & 0.04 & 14 & 7 & 7 & 0.02 \\
\hline $\mathrm{N}$ & 50 & 44 & 6 & & 50 & 40 & 10 & \\
\hline \multicolumn{9}{|c|}{ Preoperative chemotherapy } \\
\hline $\mathrm{Y}$ & 14 & 12 & 2 & 0.74 & 14 & 12 & 2 & 0.22 \\
\hline $\mathrm{N}$ & 50 & 41 & 9 & & 42 & 29 & 13 & \\
\hline Unknown & & & & & 8 & & & \\
\hline \multicolumn{9}{|l|}{ Ki67 index } \\
\hline$>50 \%$ & 8 & 7 & 1 & 0.68 & 8 & 3 & 5 & 0.02 \\
\hline$<50 \%$ & 54 & 44 & 10 & & 51 & 40 & 11 & \\
\hline Unknown & 2 & & & & 5 & & & \\
\hline
\end{tabular}

*Two-tailed $\chi^{2}$ test.

\section{Relative Quantification by Real-Time RT-PCR Analysis}

Real-time quantitative PCR amplification of the cDNA template corresponding to $20 \mathrm{ng}$ of total RNA was performed using TaqMan Universal PCR Master Mix (Applied Biosystems) in an ABI PRISM 7900 (Applied Biosystems). PCR conditions were $95^{\circ} \mathrm{C}$ for $10 \mathrm{~min}$ followed by 40 cycles of $95^{\circ} \mathrm{C}$ for $15 \mathrm{~s}$ and $60^{\circ} \mathrm{C}$ for $1 \mathrm{~min}$. eIF6- (assay ID Hs00158272_m1), Dicer-1- (assay ID 00229023_m1) and Drosha (assay ID 00203008_m1)-specific TaqMan probes were available as Assays-on-Demand from Applied Biosystems. Assays were gene dose corrected using CDKN-1B as endogenous control as previously described,$^{23}$ and normalized to 22 cases of normal whole ovary.

Data analysis was performed using Real-Time StatMiner $^{\mathrm{TM}}$ software from Integromics ${ }^{\mathrm{TM}}$ (www. integromics.com). Fold changes were calculated on filtered and quantile normalized data using the $\Delta \Delta C_{\mathrm{T}}$ method. $P$-values were calculated using a $t$-test.

\section{Immunohistochemical Stains and Statistical Analysis}

Sections $(4 \mu \mathrm{m})$ of the ovarian serous carcinoma tissue array were cut and mounted on glass slides. For antigen unmasking, deparaffinized sections were boiled (eIF6) or microwaved (Dicer, Ki-67) in 
citrate buffer $(10 \mathrm{mmol} / \mathrm{l}$ sodium citrate buffer $(\mathrm{pH}$ 6.0)) before incubation with primary antibodies. eIF6 (BD Biosciences, San Jose, CA, USA) and Dicer (Abcam, Cambridge, MA, USA) protein levels were examined using mouse monoclonal antibodies at dilutions of 1:200 and 1:50, respectively.

Ki-67 (VectorLab) protein levels were examined using rabbit polyclonal antibody at a dilution of 1:2000. Antibody staining was performed using the Biogenex Super Sensitive ${ }^{\mathrm{TM}}$ Link-Label IHC Detection system.

We recorded the intensity of eIF6 and Dicer immunoreactivity (Figure 1) in the neoplastic glands for every specimen (staining was diffuse and homogeneous in each specimen). The intensity of staining was graded on a scale from 0 to 3 : ' 0 ' reflected a lack of immunoreactivity, ' 1 ' reflected weak immunoreactivity, '2' reflected moderate immunoreactivity and ' 3 ' reflected strong homogeneous nucleocytoplasmic (eIF6) or cytoplasmic (Dicer) staining. ' 0 ' and ' 1 ' were the most common pattern of staining in normal ovarian surface epithelium. The percentage of positive cells for Ki-67 in the neoplastic glands was recorded for each specimen. Scores from all cores from one case were averaged. Two pathologists (RF and CB) scored the stained slides. All statistical analysis of immunohistochemical studies was performed with both Analyse$\mathrm{It}^{\mathrm{TM}}$ Software Ltd and MedCalc ${ }^{\mathrm{TM}}$ Software Ltd. Two sample comparisons were performed with the MannWhitney rank sum test. The association between protein and mRNA data was assessed using Pearson's correlation coefficient.

A histogram of the expression of eIF6 and Dicer showed frequency distributions with two prominent peaks at $\log _{10}$ values when both eIF6 and Dicer distribution thresholds were set at 0.2 of the $\log _{10}$ ratio (corresponding to an immunoreactivity score of 1.6) (Figure 2). Patients could be divided clearly and consistently into two groups with low and high expression. A $t$-test validated this cutoff value for separating two characteristic groups in terms of gene expression.

\section{Results}

\section{Real-Time Quantitative RT-PCR Analysis of microRNA Machinery in Ovarian Serous Adenocarcinoma}

We used real-time RT-PCR analysis to examine 50 ovarian serous adenocarcinoma cases for eIF6, Drosha and Dicer expression. All three components involved in microRNA biogenesis demonstrated upregulation in ovarian serous adenocarcinomas vs normal whole ovary (Figure 3 ).

eIF6 was upregulated 2.9-fold in ovarian serous adenocarcinoma $(P=0.0001)$. Drosha was upregulated 2.7-fold in ovarian serous adenocarcinoma $(P=0.0005)$. In addition, Dicer was upregulated 1.6-fold and had a tendency towards significance $(P=0.054)$.

\section{Immunohistochemical Analysis of eIF6 and Dicer Expression in Ovarian Serous Adenocarcinoma}

Our next investigation was to examine the protein expression levels of eIF6 and Dicer and to see if there was any correlation between the two. This was undertaken as Dicer is involved in mature
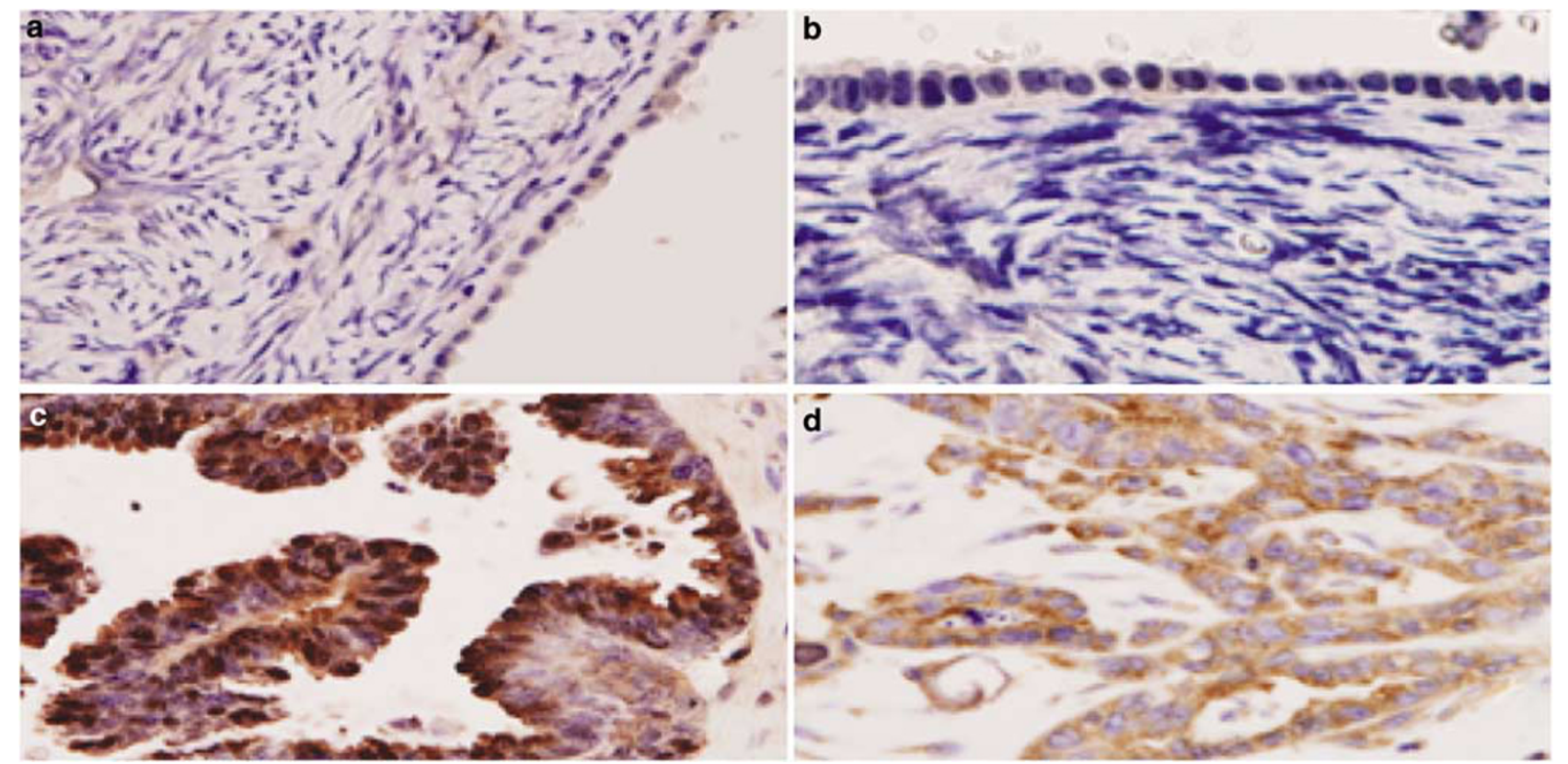

Figure 1 Expression of eIF6 and Dicer in normal ovarian surface epithelium and ovarian serous adenocarcinoma $(\times 40)$ : no staining $(0)$ for eIF6 (a) and Dicer (b) in normal ovarian surface epithelium. Strong (3) staining for eIF6 (c) and Dicer (d) in ovarian serous adenocarcinoma. 
HISTOGRAM OF THE LOG 10 PROTEIN EXPRESSION OF EIF6

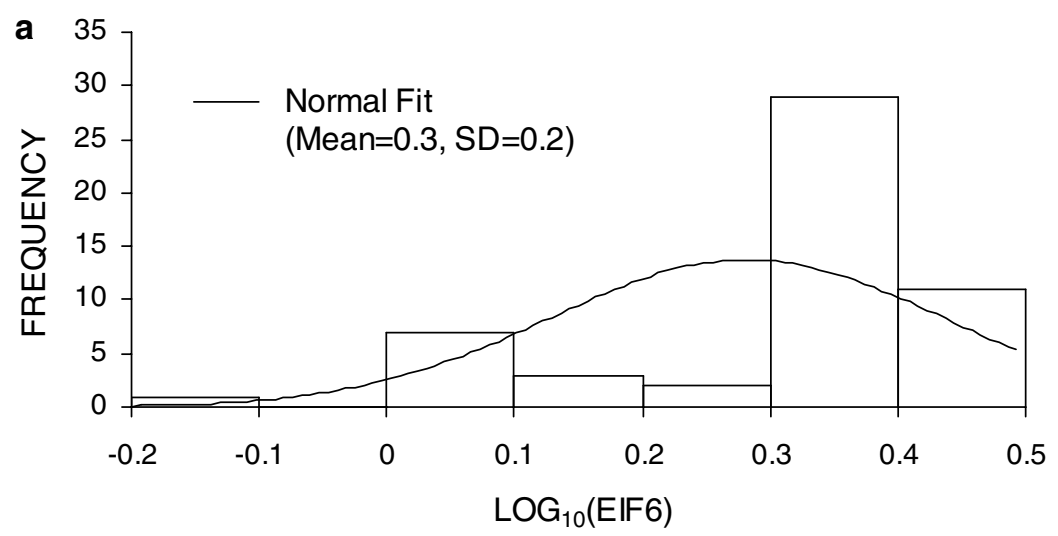

HISTOGRAM OF THE LOG 10 PROTEIN EXPRESSION OF DICER

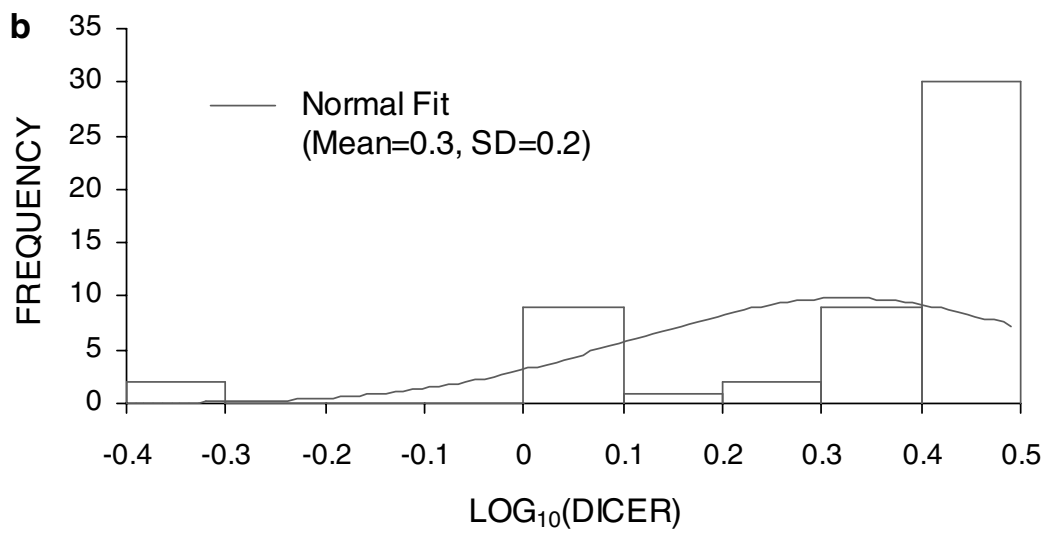

Figure 2 Histogram of the logarithmic protein expression of eIF6 (a) and Dicer (b) showing frequency distributions with two prominent peaks at $\log _{10}$ values with the thresholds set at $<0.2$ for low expression and $>0.2$ for high expression.

microRNA biogenesis and eIF6 is an effector in mature microRNA function.

Normal ovarian surface epithelium was predominantly non-reactive or showed diffuse weak nucleocytoplasmic staining for eIF6. Seventy-five percent of normal ovarian surface epithelium samples (30 of 40) showed immunoreactivity of $\leq 1.0$, and the mean eIF6 immunoreactivity in all normal ovarian surface epithelium samples $(n=40)$ was 0.94 .

In ovarian serous adenocarcinoma specimens, the mean eIF6 immunoreactivity was 2.05, a greater than two-fold increase over normal ovarian surface epithelium. Twenty percent (13/66) of samples had an immunoreactivity score of $\leq 1.6$. The majority of the ovarian serous adenocarcinoma specimens showed immunoreactivity of $\geq 1.6$ (52 of $64 ; 80 \%$ ). The difference in eIF6 immunoreactivity between normal ovarian surface epithelium and ovarian serous adenocarcinoma was statistically significant $(P<0.0001)$. The results of the immunohistochemical analysis of eIF6 expression in normal ovarian surface epithelium and ovarian serous adenocarcinoma are summarized in Table 2A.

Normal ovarian surface epithelium was predominantly non-reactive or showed diffuse weak cytoplasmic staining for Dicer. Ninety-seven percent of normal ovarian surface epithelium samples (36 of 37) showed immunoreactivity of $\leq 1.0$, and the mean Dicer immunoreactivity in all normal ovarian surface epithelium samples $(n=37)$ was 0.49 . In ovarian serous adenocarcinoma specimens, the mean Dicer immunoreactivity was 2.32, a greater than four-fold increase over normal ovarian surface epithelium. Twenty-six percent $(17 / 66)$ of samples had an immunoreactivity score of $\leq 1.6$. Seventyfour percent of ovarian serous adenocarcinoma specimens showed immunoreactivity of $\geq 1.6$ (49 of $66 ; 74 \%$ ). The difference in Dicer immunoreactivity between normal ovarian surface epithelium and ovarian serous adenocarcinoma was statistically significant $(P<0.0001)$. The results of the immunohistochemical analysis of Dicer expression in normal ovarian surface epithelium and ovarian serous adenocarcinoma are summarized in Table 2B.

A modest correlation was found between eIF6 and Dicer protein levels and mRNA expression quantified by TaqMan real-time RT-PCR $(r=0.30 ; P=0.03)$.

We found that there was no significant correlation between eIF6 protein expression levels and Dicer protein expression levels $(r=0.16, P=0.2383)$. 
MRNA EXPRESSION OF DICER, DROSHA AND EIF6 IN OVARIAN SEROUS ADENOCARCINOMA RELATIVE TO NORMAL WHOLE OVARY

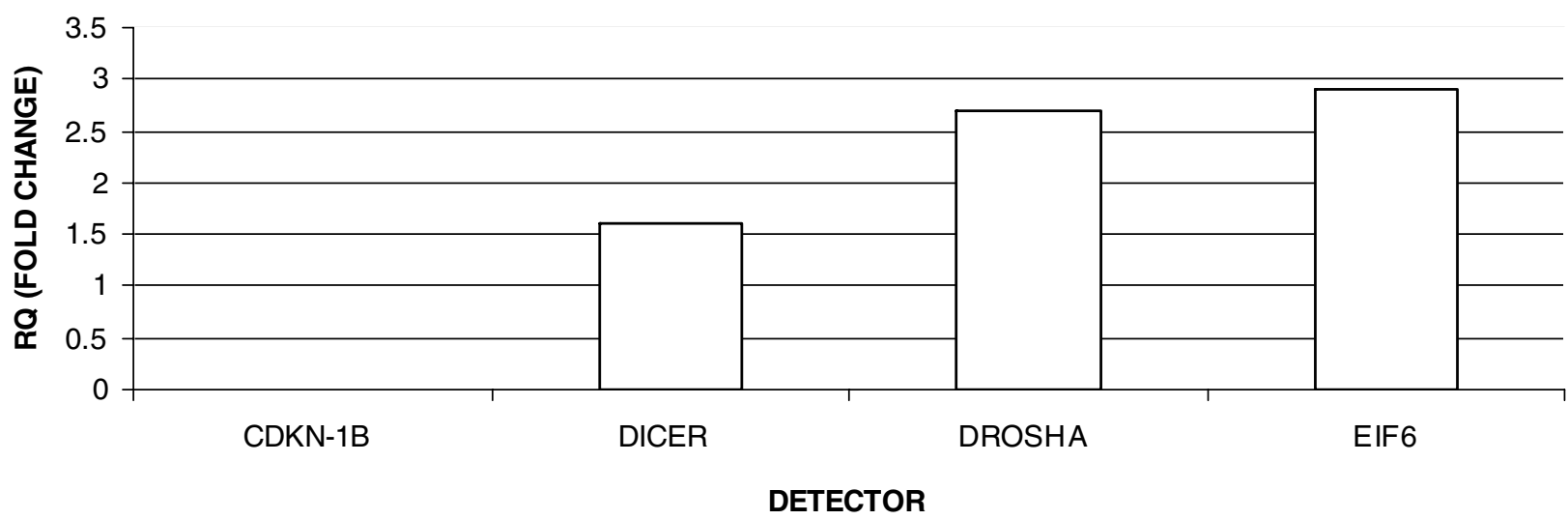

Figure 3 Mean fold changes of Dicer $(P=0.054)$, Drosha $(P=0.0005)$ and eIF6 $(P=0.0001)$ relative to normal whole ovary.

Table 2 Expression of (A) eIF6 and (B) Dicer in normal ovarian surface epithelium and ovarian serous adenocarcinoma

\begin{tabular}{|c|c|c|c|c|c|}
\hline \multirow[t]{2}{*}{ Histology } & \multirow[t]{2}{*}{ No. of cases } & \multirow[t]{2}{*}{ Mean \pm s.e.m. } & \multicolumn{3}{|c|}{ Immunoreactivity score } \\
\hline & & & \multicolumn{2}{|c|}{ Low eIF6 } & High eIF6 \\
\hline (A) Expression of eIFG $6^{\mathrm{a}}$ & & & 0 & $0.1-1.6$ & $1.7-3.0$ \\
\hline NOSE & 40 & $0.94 \pm 0.11$ & $11(27.5 \%)$ & $22(55 \%)$ & $7(17.5 \%)$ \\
\hline OSC & 65 & $2.05 \pm 0.08$ & 0 & $13(20 \%)$ & $52(80 \%)$ \\
\hline Unknown & 1 & & & & \\
\hline \multirow[t]{2}{*}{ Histology } & No. of cases & Mean \pm s.e.m. & \multicolumn{3}{|c|}{ Immunoreactivity score } \\
\hline & & & \multicolumn{2}{|c|}{ Low Dicer } & High Dicer \\
\hline (B) Expression of Dicer ${ }^{b}$ & & & 0 & $0.1-1.6$ & $1.7-3.0$ \\
\hline NOSE & 37 & $0.49 \pm 0.09$ & $20(54 \%)$ & $16(43 \%)$ & $1(3 \%)$ \\
\hline Unknown & 3 & & & & \\
\hline OSC & 66 & $2.32 \pm 0.11$ & $1(2 \%)$ & $16(24 \%)$ & $49(74 \%)$ \\
\hline
\end{tabular}

${ }^{\mathrm{a}}$ The difference in immunoreactivity between ovarian serous adenocarcinoma and normal ovarian surface epithelium and between low and high eIF6 in ovarian serous adenocarcinoma is statistically significant $(P<0.0001)$.

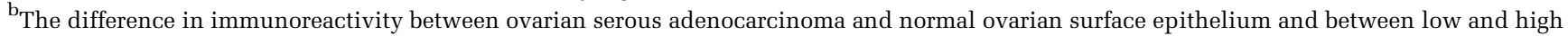
Dicer in ovarian serous adenocarcinoma is statistically significant $(P<0.0001)$.

\section{eIF6/Dicer Expression in Human Ovarian Serous Adenocarcinoma Samples Correlates with Clinicopathological Features}

Our next investigation was to consider whether protein expression levels of eIF6 and Dicer had any relationship with clinicopathological characteristics of ovarian serous adenocarcinoma.

We found that there was a statistically significant relationship between eIF6 expression levels and the presence of lymph node metastases (Table 1). Cases with high eIF6 expression showed significantly greater absence of lymph node metastases than cases with low eIF6 expression $(P=0.04)$.

There was a statistically significant relationship between Dicer expression levels and the presence of lymph node metastases. In addition, a statistically significant relationship exists between Dicer expression levels and Ki-67 proliferation index. Cases with high Dicer expression showed significantly greater absence of lymph node metastases than those with low Dicer expression $(P=0.02)$. Cases with high Dicer expression showed significantly greater prevalence of a proliferation index less than 50\% than those with low Dicer expression $(P=0.03)$.

\section{Association Between eIF6/Dicer Protein Expression and Patient Survival}

We next examined whether protein expression levels of eIF6 and Dicer were associated with patient survival after surgery. Kaplan-Meier survival curves showed that the probability of disease-free survival 

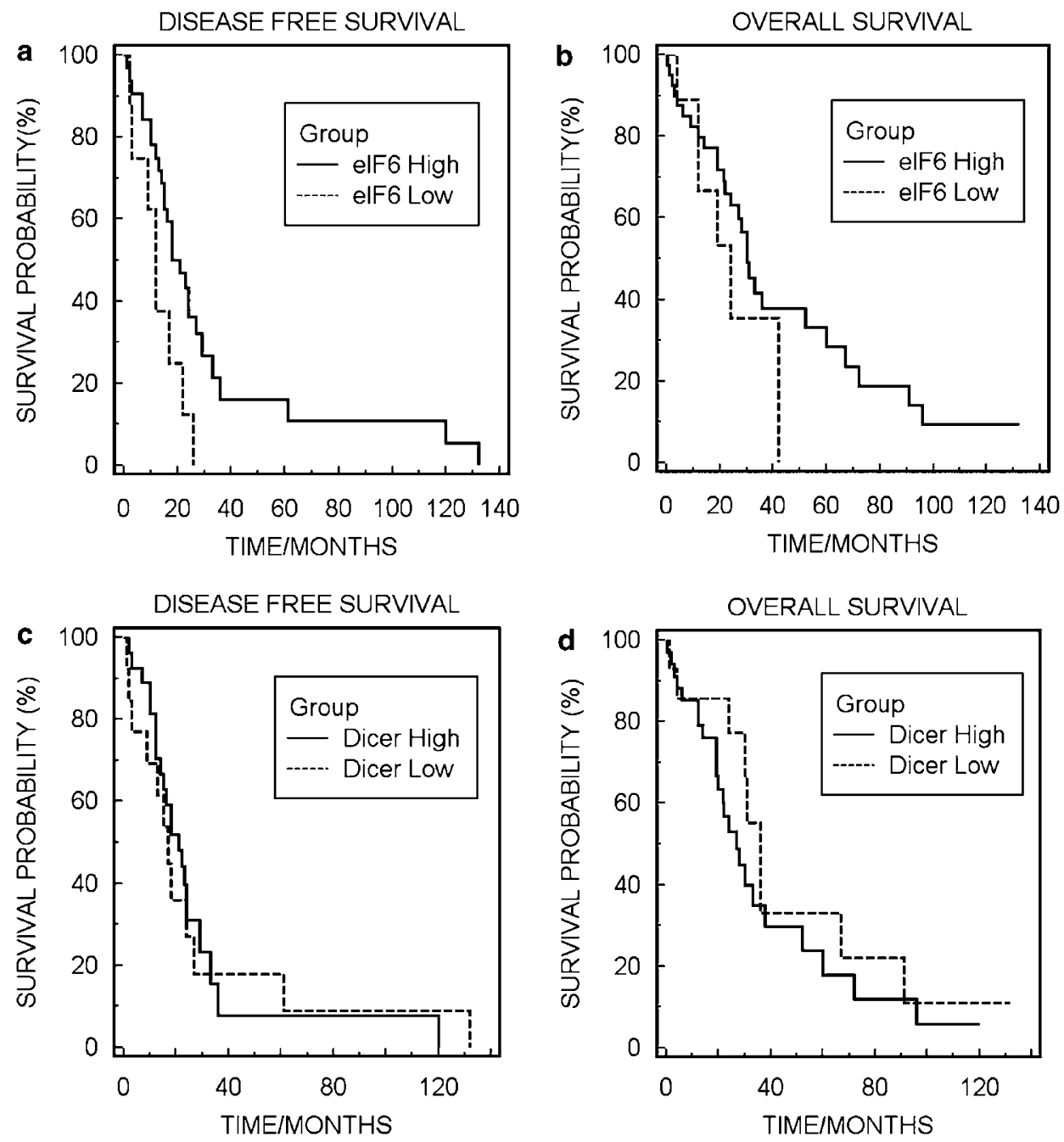

Figure 4 (a) Kaplan-Meier survival curves for ovarian serous carcinoma patients classified as showing either high or low eIF6 expression. eIF6 status was found to be associated with disease-free survival (log-rank, $P=0.02$ ). (b) eIF6 status did not exhibit a significant relationship with overall patient survival (log-rank, $P=0.22$ ). (c and d) Kaplan-Meier survival curves for ovarian serous carcinoma patients classified as showing either high or low Dicer expression. Dicer status did not exhibit a significant relationship with both disease-free and overall patient survival (log-rank, $P=0.84$ and $P=0.31$ ).

was significantly lower for patients with low levels of eIF6 expression $(P=0.02$ by log-rank test; Figure $4 \mathrm{a}$ and b). eIF6 status did not exhibit a significant relationship with overall patient survival $(P=0.22$ by log-rank test).

Kaplan-Meier survival curves showed that the probability of both disease-free and overall survival was not significantly different between patients with low and high levels of Dicer expression $(P=0.84$ and 0.31 , respectively, by log-rank test; Figure 4c and d).

The interrelationship of possible prognostic factors and disease-free survival was further analysed by means of Cox proportional hazards modelling using age, grade, disease stage, preoperative chemotherapy and presence of lymph node metastases as well as the expression levels of eIF6 and Dicer as variables. Reduced expression of eIF6 was identified as a significant and independent prognostic factor $(P=0.03)$. The hazard ratio for earlier relapse was 2.6 (95\% confidence interval: 1.10-5.97) for low vs high expression levels of eIF6. These findings indicate that the expression levels of eIF6 appear to have a significant impact on disease-free survival in ovarian serous adenocarcinoma patients.

\section{Discussion}

In this study, we evaluated the expression and clinical relevance of eIF6 and Dicer in human ovarian serous adenocarcinoma. We provide definitive evidence that eIF6 and Dicer are both upregulated in a significant proportion of ovarian serous adenocarcinoma and are associated with specific clinicopathological features, most notably low eIF6 expression being associated with reduced disease-free survival. Indeed, this association may 
be related to cases with low eIF6 expression showing significantly greater prevalence of lymph node metastases than cases with high eIF6 expression. Furthermore, Drosha, another component of the microRNA machinery, was found to be upregulated. To the best of our knowledge, this is the first report of alterations of eIF6 and microRNA machinery in human ovarian serous adenocarcinoma.

Similar to our study, overexpression of eIF6 has also been previously demonstrated in both head and neck squamous cell carcinomas and colorectal carcinomas. ${ }^{24,25}$ The mechanism of eIF6 overexpression may be due to gene amplification. Human eIF6 is mapped to chromosome 20 (20q12), and previous studies $^{26-30}$ have identified amplification of the chromosomal region 20q12-13 in ovarian cancer. Indeed, amplifications of the 20q12 region have also been found by CGH analysis in colorectal carcinomas. ${ }^{31}$ The role of upregulated eIF6 in ovarian serous adenocarcinoma may be interpreted as a consequence of increased protein turnover in rapidly proliferating malignant cells based on its role in ribosome assembly.

Recently, however, eIF6 has been linked with the regulation of the canonical WNT signalling pathway at the level of the $\beta$-catenin protein. ${ }^{32} \beta$-Catenin is important in cell adhesion and is a key molecule in the WNT signalling pathway. Oncogenes such as cyclin D1, ${ }^{33}$ c-myc, ${ }^{34}$ PPAR $\delta^{35}$ are targets of the $\mathrm{WNT} / \beta$-catenin pathway. Furthermore, eIF6 is known to interact with integrin- $\beta 4 .{ }^{36}$ Deregulation of the WNT signalling is well described in ovarian serous adenocarcinoma pathogenesis, ${ }^{37-40}$ indicating that eIF6 may have multiple biological roles in ovarian serous adenocarcinoma tumorigenesis.

Similar to our study, alterations in the expression levels of Dicer have been found in prostate adenocarcinoma and lung adenocarcinomas. ${ }^{41,42}$ Upregulation of Dicer correlated with clinical stage, lymph node status and Gleason score in prostate adenocarcinoma. ${ }^{41}$ Dicer overexpression was noted in precursor lesions of lung adenocarcinoma and varied with histological subtype of lung carcinoma. ${ }^{42}$ Furthermore, an association between reduced Dicer expression and poor prognosis has been identified in lung cancer patients. ${ }^{8}$ Interestingly, high Dicer expression correlated with proliferation index and lymph node status in our study; however, no correlation was demonstrated between Dicer expression and patient prognosis.

The mechanism for Dicer deregulation appears to be complex. Chiosea et $a l^{42}$ demonstrated that a fraction of lung adenocarcinomas lose Dicer as a result of deletions at the Dicer locus, whereas Karube et $a l^{8}$ found that hypermethylation of CpG sites in the promoter region of Dicer is absent in a subset of lung carcinomas.

Human Dicer is mapped to chromosome 14(14q32.3). In ovarian cancer, loss of heterozygosity is found at a rate of $49 \%$ at chromosome $14 q .{ }^{43}$ This is the site of the Dicer locus, indicating that the mechanism accounting for low levels of Dicer in our study maybe due to genomic instability at this region.

Recently, microRNAs were implicated in the development of ovarian cancer: 39 microRNAs were found to be differentially regulated between tumour and normal ovarian tissue. ${ }^{13}$ Specifically, in the serous histotype, 37 of 41 differentially expressed microRNAs were downregulated relative to normal ovary. However, selection of control cells may strongly influence the determination of differentially expressed genes in such ovarian cancer studies. ${ }^{44}$ Epithelial ovarian cancer is thought to arise from three proposed origins including the ovarian surface epithelium or mullerian inclusions, fallopian tube mucosa and mullerian epithelium elsewhere in the peritoneal cavity. ${ }^{45-49}$ Expression profiling studies involve the comparison of ovarian serous adenocarcinoma to normal ovarian surface epithelium. In this regard, because normal ovarian surface epithelium comprises only a small percentage of the total cells of whole normal ovary and ovarian tumour cells contain a significant amount of stromal and host-derived immune cells, normal ovarian surface epithelium is seen as an optimal control in gene expression studies. In this regard, microRNA profiles of ovarian serous adenocarcinoma relative to normal ovarian serous epithelium may differ from that published by Iorio et al. ${ }^{13}$ Furthermore, microRNA expression is both temporally and spatially heterogeneous and it is not inconceivable that microRNA profiles and indeed Dicer protein levels may differ within the same tumour type.

Little is known about the mechanisms of microRNA deregulation in neoplastic tissue. MicroRNA genes are commonly located at minimal regions of amplification, loss of heterozygosity and breakpoint regions, suggesting that abnormal microRNA profiles can be caused by somatic gene mutation. ${ }^{50}$ Recent research suggests that DNA methylation is also involved in the regulation of microRNA expression, and the methylation can be reversed by DNA methyltransferase inhibitors. ${ }^{51-54}$ Mature microRNA biogenesis, however, is dependant on Dicer and it is conceivable that lower Dicer levels may reduce microRNA expression in ovarian cancer cells. This hypothesis, however, relies on the assumption that Dicer is functional. Many research groups demonstrate a functional interfering RNA pathway in ovarian adenocarcinoma cell lines by using small interfering RNA constructs targeting mRNAs. ${ }^{55,56}$ In addition, global microRNA profiling has showed that Drosha overexpression in cervical squamous cell carcinoma appears to be of functional significance. ${ }^{57}$ However, despite evidence for decreased Dicer levels leading to a decrease in microRNA let-7, ${ }^{7}$ no definitive evidence linking global alterations in microRNA profiles to Dicer levels has been demonstrated to date. In fact, increased Dicer expression was observed in lung 
adenocarcinoma relative to normal alveolar epithelium in a tumour where global downregulation of microRNA has been reported. ${ }^{58}$ Alternate events such as somatic gene alteration and epigenetic regulation need to be pursued.

In conclusion, we provide definitive evidence that eIF6 and Dicer are both upregulated in a significant proportion of ovarian serous adenocarcinomas and are associated with specific clinicopathological features of ovarian serous adenocarcinoma, most notably low eIF6 expression being associated with reduced disease-free survival. The status of eIF6 and proteins of the microRNA machinery may help predict toxicity and susceptibility to future interfering RNA-based therapy.

\section{Disclosure/conflict of interest}

Dr Richard Flavin is funded by a HRB Ireland Clinical Research Fellowship. The authors have no conflict of interest to disclose.

\section{References}

1 Hoskins WJ. Prospective on ovarian cancer: why prevent? J Cell Biochem Suppl 1995;23:189-199.

2 Parkin DM, Bray F, Ferlay J, et al. Global cancer statistics, 2002. CA Cancer J Clin 2005;55:74-108.

3 Scully RE. Classification of human ovarian tumors. Environ Health Perspect 1987;73:15-24.

4 Kikkawa F, Nawa A, Ino K, et al. Advances in treatment of epithelial ovarian cancer. Nagoya J Med Sci 2006; 68:19-26.

5 Calin GA, Dumitru CD, Shimizu M, et al. Frequent deletions and down-regulation of microRNA genes miR15 and miR16 at 13q14 in chronic lymphocytic leukemia. Proc Natl Acad Sci USA 2002; 99:15524-15529.

6 Michael MZ, SM OC, van Holst Pellekaan NG, et al. Reduced accumulation of specific microRNAs in colorectal neoplasia. Mol Cancer Res 2003;1: 882-891.

7 Takamizawa J, Konishi $\mathrm{H}$, Yanagisawa $\mathrm{K}$, et al. Reduced expression of the let-7 microRNAs in human lung cancers in association with shortened postoperative survival. Cancer Res 2004;64:3753-3756.

8 Karube Y, Tanaka H, Osada $\mathrm{H}$, et al. Reduced expression of Dicer associated with poor prognosis in lung cancer patients. Cancer Sci 2005;96:111-115.

9 Hayashita Y, Osada H, Tatematsu Y, et al. A polycistronic microRNA cluster, miR-17-92, is overexpressed in human lung cancers and enhances cell proliferation. Cancer Res 2005;65:9628-9632.

10 Ambros V. The functions of animal microRNAs. Nature 2004;431:350-355.

11 Bartel DP. MicroRNAs: genomics, biogenesis, mechanism, and function. Cell 2004;116:281-297.

$12 \mathrm{Lu}$ J, Getz G, Miska EA, et al. MicroRNA expression profiles classify human cancers. Nature 2005;435: 834-838.

13 Iorio MV, Visone R, Di Leva G, et al. MicroRNA signatures in human ovarian cancer. Cancer Res 2007; 67:8699-8707.
14 Ambros V. MicroRNA pathways in flies and worms: growth, death, fat, stress, and timing. Cell 2003;113: 673-676.

15 Grosshans H, Slack FJ. Micro-RNAs: small is plentiful. J Cell Biol 2002;156:17-21.

16 Lee Y, Jeon K, Lee JT, et al. MicroRNA maturation: stepwise processing and subcellular localization. EMBO J 2002;21:4663-4670.

17 Gregory RI, Shiekhattar R. MicroRNA biogenesis and cancer. Cancer Res 2005;65:3509-3512.

18 Ceci M, Gaviraghi C, Gorrini C, et al. Release of eIF6 (p27BBP) from the 60S subunit allows $80 \mathrm{~S}$ ribosome assembly. Nature 2003;426:579-584.

19 Russell DW, Spremulli LL. Purification and characterization of a ribosome dissociation factor (eukaryotic initiation factor 6) from wheat germ. J Biol Chem 1979; 254:8796-8800.

20 Valenzuela DM, Chaudhuri A, Maitra U. Eukaryotic ribosomal subunit anti-association activity of calf liver is contained in a single polypeptide chain protein of $\mathrm{Mr}=25,500$ (eukaryotic initiation factor 6). J Biol Chem 1982;257:7712-7719.

21 Raychaudhuri P, Stringer EA, Valenzuela DM, et al. Ribosomal subunit antiassociation activity in rabbit reticulocyte lysates. Evidence for a low molecular weight ribosomal subunit antiassociation protein factor $(\mathrm{Mr}=25,000) . \quad \mathrm{J}$ Biol Chem 1984;259: 11930-11935.

22 Chendrimada TP, Finn KJ, Ji X, et al. MicroRNA silencing through RISC recruitment of eIF6. Nature 2007;447:823-828.

23 Denning KM, Smyth PC, Cahill SF, et al. A molecular expression signature distinguishing follicular lesions in thyroid carcinoma using preamplification RT-PCR in archival samples. Mod Pathol 2007;20:1095-1102.

24 Rosso P, Cortesina G, Sanvito F, et al. Overexpression of p27BBP in head and neck carcinomas and their lymph node metastases. Head Neck 2004;26:408-417.

25 Sanvito F, Vivoli F, Gambini S, et al. Expression of a highly conserved protein, p27BBP, during the progression of human colorectal cancer. Cancer Res 2000;60: 510-516.

26 Tanner MM, Grenman S, Koul A, et al. Frequent amplification of chromosomal region 20q12-q13 in ovarian cancer. Clin Cancer Res 2000;6: 1833-1839.

27 Sonoda G, Palazzo J, du Manoir S, et al. Comparative genomic hybridization detects frequent overrepresentation of chromosomal material from 3q26, 8q24, and $20 q 13$ in human ovarian carcinomas. Genes Chromosomes Cancer 1997;20:320-328.

28 Arnold N, Hagele L, Walz L, et al. Overrepresentation of $3 q$ and $8 q$ material and loss of $18 q$ material are recurrent findings in advanced human ovarian cancer. Genes Chromosomes Cancer 1996;16:46-54.

29 Iwabuchi H, Sakamoto M, Sakunaga H, et al. Genetic analysis of benign, low-grade, and high-grade ovarian tumors. Cancer Res 1995;55:6172-6180.

30 Tapper J, Sarantaus L, Vahteristo P, et al. Genetic changes in inherited and sporadic ovarian carcinomas by comparative genomic hybridization: extensive similarity except for a difference at chromosome 2q24-q32. Cancer Res 1998;58:2715-2719.

31 Liu XP, Kawauchi S, Oga A, et al. Chromosomal aberrations detected by comparative genomic hybridization predict outcome in patients with colorectal carcinoma. Oncol Rep 2007;17:261-267. 
32 Ji Y, Shah S, Soanes K, et al. Eukaryotic initiation factor 6 selectively regulates Wnt signaling and betacatenin protein synthesis. Oncogene 2008;27:755-762.

33 Tetsu O, McCormick F. Beta-catenin regulates expression of cyclin D1 in colon carcinoma cells. Nature 1999;398:422-426.

34 He TC, Sparks AB, Rago C, et al. Identification of cMYC as a target of the APC pathway. Science 1998; 281:1509-1512.

35 He TC, Chan TA, Vogelstein B, et al. PPARdelta is an APC-regulated target of nonsteroidal anti-inflammatory drugs. Cell 1999;99:335-345.

36 Biffo S, Sanvito F, Costa S, et al. Isolation of a novel beta4 integrin-binding protein (p27(BBP)) highly expressed in epithelial cells. J Biol Chem 1997;272: 30314-30321.

37 Sarrio D, Moreno-Bueno G, Sanchez-Estevez C, et al. Expression of cadherins and catenins correlates with distinct histologic types of ovarian carcinomas. Hum Pathol 2006;37:1042-1049.

38 Kildal W, Risberg B, Abeler VM, et al. beta-catenin expression, DNA ploidy and clinicopathological features in ovarian cancer: a study in 253 patients. Eur J Cancer 2005;41:1127-1134.

39 Shedden KA, Kshirsagar MP, Schwartz DR, et al. Histologic type, organ of origin, and Wnt pathway status: effect on gene expression in ovarian and uterine carcinomas. Clin Cancer Res 2005;11: 2123-2131.

40 Lee CM, Shvartsman H, Deavers MT, et al. Beta-catenin nuclear localization is associated with grade in ovarian serous carcinoma. Gynecol Oncol 2003;88:363-368.

41 Chiosea S, Jelezcova E, Chandran U, et al. Upregulation of dicer, a component of the MicroRNA machinery, in prostate adenocarcinoma. Am J Pathol 2006;169:1812-1820.

42 Chiosea S, Jelezcova E, Chandran U, et al. Overexpression of Dicer in precursor lesions of lung adenocarcinoma. Cancer Res 2007;67:2345-2350.

43 Bandera CA, Takahashi H, Behbakht K, et al. Deletion mapping of two potential chromosome 14 tumor suppressor gene loci in ovarian carcinoma. Cancer Res 1997;57:513-515.

44 Zorn KK, Jazaeri AA, Awtrey CS, et al. Choice of normal ovarian control influences determination of differentially expressed genes in ovarian cancer expression profiling studies. Clin Cancer Res 2003;9: 4811-4818.
45 Feeley KM, Wells M. Precursor lesions of ovarian epithelial malignancy. Histopathology 2001;38:87-95.

46 Crum CP, Drapkin R, Kindelberger D, et al. Lessons from BRCA: the tubal fimbria emerges as an origin for pelvic serous cancer. Clin Med Res 2007;5:35-44.

47 Crum CP, Drapkin R, Miron A, et al. The distal fallopian tube: a new model for pelvic serous carcinogenesis. Curr Opin Obstet Gynecol 2007;19:3-9.

48 Dalrymple JC, Bannatyne P, Russell P, et al. Extraovarian peritoneal serous papillary carcinoma. A clinicopathologic study of 31 cases. Cancer 1989;64:110-115.

49 Kindelberger DW, Lee Y, Miron A, et al. Intraepithelial carcinoma of the fimbria and pelvic serous carcinoma: evidence for a causal relationship. Am J Surg Pathol 2007;31:161-169.

50 Zhang L, Huang J, Yang N, et al. microRNAs exhibit high frequency genomic alterations in human cancer. Proc Natl Acad Sci USA 2006;103:9136-9141.

$51 \mathrm{Lu} \mathrm{L}$, Katsaros D, de la Longrais IA, et al. Hypermethylation of let-7a-3 in epithelial ovarian cancer is associated with low insulin-like growth factor-II expression and favorable prognosis. Cancer Res 2007; 67:10117-10122.

52 Lujambio A, Ropero S, Ballestar E, et al. Genetic unmasking of an epigenetically silenced microRNA in human cancer cells. Cancer Res 2007;67:1424-1429.

53 Brueckner B, Stresemann C, Kuner R, et al. The human let-7a-3 locus contains an epigenetically regulated microRNA gene with oncogenic function. Cancer Res 2007;67:1419-1423.

54 Saito Y, Liang G, Egger G, et al. Specific activation of microRNA-127 with downregulation of the protooncogene BCL6 by chromatin-modifying drugs in human cancer cells. Cancer Cell 2006;9:435-443.

55 Fan Y, Xin XY, Chen BL, et al. Knockdown of RAB25 expression by RNAi inhibits growth of human epithelial ovarian cancer cells in vitro and in vivo. Pathology 2006;38:561-567.

56 El-Naggar SM, Malik MT, Kakar SS. Small interfering RNA against PTTG: a novel therapy for ovarian cancer. Int J Oncol 2007;31:137-143.

57 Muralidhar B, Goldstein LD, Ng G, et al. Global microRNA profiles in cervical squamous cell carcinoma depend on Drosha expression levels. J Pathol 2007;212:368-377.

58 Yanaihara N, Caplen N, Bowman E, et al. Unique microRNA molecular profiles in lung cancer diagnosis and prognosis. Cancer Cell 2006;9:189-198. 